hep-ph/0610277

SLAC-PUB-12168

TU-778

October, 2006

\title{
Possible Signals of Wino LSP at the Large Hadron Collider
}

\author{
M. Ibe ${ }^{1}$, Takeo Moroi ${ }^{2}$ and T. T. Yanagida ${ }^{3,4}$ \\ ${ }^{1}$ Stanford Linear Accelerator Center, Stanford University, \\ Stanford, CA94309 \\ ${ }^{2}$ Department of Physics, Tohoku University, \\ Sendai, Japan \\ ${ }^{3}$ Department of Physics, University of Tokyo, \\ Tokyo 113-0033, Japan \\ ${ }^{4}$ Research Center for the Early Universe, University of Tokyo, \\ Tokyo 113-0033, Japan
}

\begin{abstract}
We consider a class of anomaly-mediated supersymmetry breaking models where gauginos acquire masses mostly from anomaly mediation while masses of other superparticles are from Kähler interactions, which are as large as gravitino mass $\sim \mathcal{O}(10-100) \mathrm{TeV}$. In this class of models, the neutral Wino becomes the lightest superparticle in a wide parameter region. The mass splitting between charged and neutral Winos are very small and experimental discovery of such Winos is highly non-trivial. We discuss how we should look for Wino-induced signals at Large Hadron Collider.
\end{abstract}




\section{Introduction}

In the vacua of broken supersymmetry (SUSY) not only the gravitino but also squarks and sleptons acquire tree-level SUSY-breaking masses through supergravity (SUGRA) effects [1]. On the other hand, if there is no singlet field in the hidden sector, quantumloop corrections generate gaugino masses, which is often called as anomaly mediation [2, 3. This is the most economical mechanism for giving SUSY-breaking masses to all SUSY particles in the SUSY standard model (SSM) since it does not require any extra field other than ones responsible for the SUSY breaking. In this model the gauginos are much lighter than the squarks and sleptons due to the loop effects. That is, there is a little disparity in the spectrum of SUSY particles. The masses of squarks and sleptons (and also that of the gravitino) are $\mathcal{O}(10-100) \mathrm{TeV}$ for the gaugino masses less than $\mathcal{O}(1) \mathrm{TeV}$. Because of this disparity the anomaly mediation model is free from all problems in the SSM such as flavor-changing neutral current and CP problems [4. Furthermore, the mass of the lightest Higgs boson is naturally above the experimental lower bound $m_{H}>114.4$ $\mathrm{GeV}$ [5]. From the cosmological point of view, the gravitino-overproduction problem [6] in the early universe is much less serious in the above model due to the relatively large gravitino mass. It is also notable that a consistent scenario of thermal leptogenesis [7] is possible in the present scheme [8], and that the lightest superparticle (LSP), which is the neutral Wino, can be a good candidate for the dark matter in the universe [9, 10].

The purpose of this paper is to discuss possible tests of the above anomaly mediation model at the Large Hadron Collider (LHC) experiments. The crucial point is that the charged and neutral Winos are the most-motivated LSPs whose masses almost degenerate with each other, and the neutral Wino $\tilde{W}^{0}$ is slightly lighter than the charged one $\tilde{W}^{ \pm}$ [11]. In a cosmological scenario [10], a sizable amount of Winos are produced by the decay of gravitino originating from inflaton decay [12, and provided that such Wino can be the dark matter in the universe, the mass of the Wino is predicted as $M_{2} \simeq 100 \mathrm{GeV}-2 \mathrm{TeV}$. Therefore, it is highly possible that the Wino is within the reach of the LHC. ${ }^{1}$

\footnotetext{
${ }^{1}$ The thermally produced Winos cannot explain the observed dark matter density unless it is very heavy, $M_{2} \simeq 2 \mathrm{TeV}$, which requires gravitino with mass $m_{3 / 2}=\mathcal{O}(1) \mathrm{PeV}[13$.
} 
Unfortunately, however, it is also possible that superparticles other than Winos can be hardly produced at the LHC, depending on the model parameters. Indeed, as we will see, the gluino-Wino mass ratio $M_{3} / M_{2}$ can be larger than the prediction of the pure anomaly mediation model, $M_{3} / M_{2} \simeq 8$. Thus, it is difficult to produce the gluinos at the LHC except for very optimistic cases as well as sfermions. Therefore it is now highly important to discuss signals of the Wino production at the LHC.

\section{Properties of Winos}

We first discuss the mass spectrum of superparticles. We assume that the masses of squarks, sleptons and Higgs bosons are dominantly from SUGRA effects. With a generic Kähler potential, all the scalar bosons except the lightest Higgs boson acquire SUSY breaking masses of the order of the gravitino mass $\left(m_{3 / 2}\right)$. For the gaugino masses, on the contrary, tree-level SUGRA contributions are extremely suppressed if there is no singlet elementary field in the hidden sector. In this paper, we adopt the above setup and consider the situation that the scalar masses (as well as the gravitino mass) are of the order of $\mathcal{O}(10-100) \mathrm{TeV}$ while gauginos are much lighter. In addition, we also assume that Higgsinos and heavy Higgs bosons have masses of $\mathcal{O}(10-100) \mathrm{TeV}$. Hereafter, we call such a scenario as "no singlet scenario".

If there is no singlet field in the hidden sector, effects of anomaly mediation becomes very important in generating gaugino masses [2, 3. At $Q \sim m_{3 / 2}$ (with $Q$ being a renormalization scale), anomaly-mediation contributions to the gaugino masses are given by

$$
M_{a}^{(A M S B)}=-\frac{b_{a} g_{a}^{2}}{16 \pi^{2}} m_{3 / 2},
$$

where $a$ runs in three standard-model gauge groups $(a=1,2,3), g_{a}$ denote gauge coupling constants, and $b_{a}$ coefficients of the renormalization-group equations for $g_{a}$, i.e., $b_{a}=$ $(-33 / 5,-1,3)$.

The important point is that the natural sizes of so-called $\mu$ - and $B$-parameters for Higgs multiplets (as well as their SUSY breaking masses) are as large as the gravitino 
mass in the "no singlet scenario". Indeed, we expect the following Kähler potential:

$$
K \ni c H_{u} H_{d}+\frac{c^{\prime}}{M_{G}^{2}} X^{\dagger} X H_{u} H_{d}+h . c .
$$

where $X$ denotes a chiral superfield in the hidden sector, which may be elementary or composite, $M_{G}$ is the reduced Planck scale, and $c$ and $c^{\prime}$ are coefficients of $\mathcal{O}(1)$. Then, the $\mu$ - and the $B$-parameters are given by $[14]^{2}$

$$
\begin{aligned}
\mu & =c m_{3 / 2}, \\
B \mu & =c m_{3 / 2}^{2}+c^{\prime} \frac{\left|F_{X}\right|^{2}}{M_{G}^{2}},
\end{aligned}
$$

where $F_{X}$ is the vacuum expectation value of the $F$-component of $X$. Thus, $\mu$ - and $B$-parameters are both of $\mathcal{O}\left(m_{3 / 2}\right)$ and they are independent. For the successful electroweak symmetry breaking, one linear combination of Higgs bosons should become light: with the so-called $\beta$-parameter, the (standard-model-like) light doublet is denoted as $\sin \beta H_{u}-\cos \beta H_{d}^{*}$, where $H_{u}$ and $H_{d}$ are up- and down-type Higgs bosons, respectively.

Then, threshold corrections to the gaugino masses from the Higgs-Higgsino loop are given by [3, 15]

$$
\begin{aligned}
\Delta M_{1}^{(\text {Higgs })} & =\frac{3}{5} \frac{g_{1}^{2}}{16 \pi^{2}} L, \\
\Delta M_{2}^{(\text {Higgs })} & =\frac{g_{2}^{2}}{16 \pi^{2}} L, \\
\Delta M_{3}^{(\text {Higgs })} & =0,
\end{aligned}
$$

where

$$
L \equiv \mu \sin 2 \beta \frac{m_{A}^{2}}{|\mu|^{2}-m_{A}^{2}} \ln \frac{|\mu|^{2}}{m_{A}^{2}} .
$$

Here, $m_{A}$ is the mass of heavy Higgs bosons. When $\mu=\mathcal{O}\left(m_{3 / 2}\right), \Delta M_{a}^{(\text {Higgs })}$ (for $\left.a=1,2\right)$ and $M_{a}^{(A M S B)}$ are of the same order, and hence we have sizable deviations from the relations in Eq. (11) of the pure anomaly mediation.

\footnotetext{
${ }^{2}$ We assume that the vacuum expectation value of $X$ is much smaller than $M_{G}$.
} 


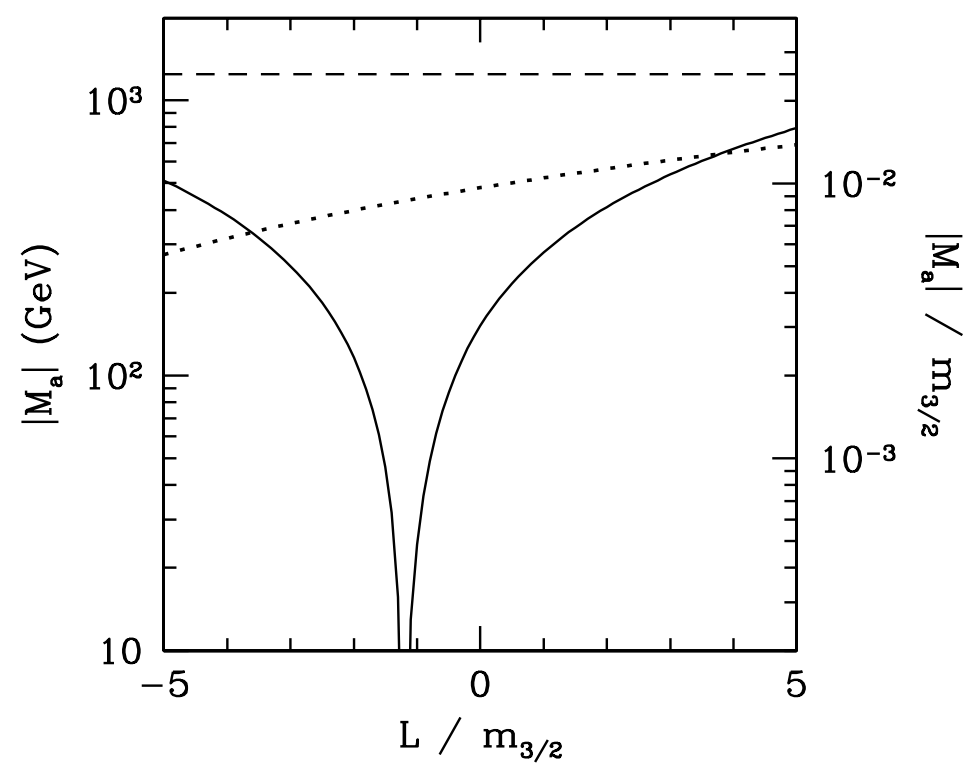

Figure 1: Gaugino masses as functions of $L / m_{3 / 2}$ for a real value of $L$. The Wino mass is given in the solid line, while the dotted and dashed lines are for Bino and gluino masses, respectively. Here we take $m_{3 / 2}=50 \mathrm{TeV}$. The cancellation of the Wino mass for $L / m_{3 / 2} \simeq-1$ is a result of our specific choice of the phase, $\operatorname{Arg}(L)=\pi$. For a generic phase of $L$, the cancellation becomes milder, though the Wino mass can be much lighter than $M_{2}^{(A M S B)}$ for $\left|L / m_{3 / 2}\right| \simeq 1$ and $\operatorname{Arg}(L) \sim \pi$.

The $\mu$-parameter (and $L$ ) is a complex variable and the Wino and Bino masses depend on the relative phase between $\mu$ and $m_{3 / 2}$. (Here, we use the bases where the gravitino mass is real and positive.) Importantly, with a distractive interference between $M_{2}^{(A M S B)}$ and $\Delta M_{2}^{(\text {Higgs })}$, the Wino mass can be even smaller than the purely anomaly mediated one. As an example, in Fig. 1. we plot the gaugino masses for $m_{3 / 2}=50 \mathrm{TeV}$ as functions of $L / m_{3 / 2}$, assuming that $L$ is real for simplicity. In this case, we see that the threshold corrections drastically change the gaugino mass and even cancel the anomaly mediated Wino mass. In our analysis, gaugino masses are given by $M_{a}=M_{a}^{(A M S B)}+\Delta M_{a}^{(\text {Higgs })}$ at $Q=m_{3 / 2}$, and we take into account the effects of renormalization group evolutions below this scale. We have checked that the ratios of two gaugino masses are insensitive to the gravitino mass for a given $L / m_{3 / 2}$.

A wide variety of gaugino mass in the "no singlet scenario" has strong implications for 
collider physics. In the pure anomaly mediation, all the gaugino masses are determined only by the gravitino mass and corresponding gauge coupling constants as shown in Eq. (1), and hence a negative search for gluino-induced signals results in lower bounds on other gaugino masses, in particular, that of Wino mass. In the "no singlet scenario", on the contrary, gluino and Wino masses are independent. Thus, even if gluino-induced signals will not be found at the LHC, we will still have a strong motivation to look for Wino-induced signals for the parameter region where Wino is relatively light.

In the pure anomaly mediation model, the lightest gaugino is always the Wino. However, in the "no singlet scenario", the Wino LSP is not a generic consequence any more since the Bino may become lighter than Wino. However, Fig. 1 shows that Wino becomes the LSP if $\left|L / m_{3 / 2}\right|$ is less than a few, which is the case when $|\mu|$ and $m_{A}$ are both fairly close to the gravitino mass. In addition, the Wino LSP scenario can never cease from being the most motivated scenario from the point of view of cosmology. This is because the Bino LSP results in the overclosure of the universe [3]. Therefore, in this paper, we concentrate on the Wino LSP scenario, where the lightest neutralino and the lightest chargino are both (almost) purely Winos.

Now, we consider detailed properties of the Wino. As discussed in Ref. [11, the dominant mass splitting between the charged and neutral Winos comes from one-loop gauge boson contributions to the gaugino masses when $\mu$ is large. The splitting is given by

$$
\Delta M=m_{\tilde{W}^{ \pm}}-m_{\tilde{W}^{0}}=\frac{g_{2}^{2}}{16 \pi^{2}} M_{2}\left[f\left(r_{W}\right)-\cos ^{2} \theta_{W} f\left(r_{Z}\right)-\sin ^{2} \theta_{W} f(0)\right],
$$

where $f(r)=\int_{0}^{1} d x\left(2+2 x^{2}\right) \ln \left[x^{2}+(1-x) r^{2}\right]$ and $r_{i}=m_{i} / M_{2}$. Requiring that the charged Wino mass be larger than the current experimental bound $M_{2} \geq 88 \mathrm{GeV}$ [16], the mass difference is in the range $155 \mathrm{MeV} \lesssim \Delta M \lesssim 170 \mathrm{MeV}$. Then, the dominant decay mode of charged Wino is given by $\tilde{W}^{ \pm} \rightarrow \tilde{W}^{0} \pi^{ \pm}$; the decay rate of this process is

$$
\Gamma\left(\tilde{W}^{ \pm} \rightarrow \tilde{W}^{0} \pi^{ \pm}\right)=\frac{2 G_{F}^{2}}{\pi} \cos ^{2} \theta_{c} f_{\pi}^{2} \Delta M^{3}\left(1-\frac{m_{\pi}^{2}}{\Delta M^{2}}\right)^{1 / 2},
$$

where $f_{\pi} \simeq 130 \mathrm{MeV}$, and $\theta_{c}$ is the Cabbibo angle. Notice that the three-body decay processes $\tilde{W}^{ \pm} \rightarrow \tilde{W}^{0} l^{ \pm} \nu_{l}$ are negligible in our study; branching ratios of this type of 
processes are a few $\%$ or smaller for $M_{2} \gtrsim 88 \mathrm{GeV}^{3}$ We also estimate the lifetime of charged Wino, which is given by $\mathcal{O}\left(10^{-10}\right)$ sec. Then, charged Winos produced at the LHC travel typically $\mathcal{O}(1-10) \mathrm{cm}$ before they decay. As we discuss in the following, this fact has very important consequences in the study of our scenario at the LHC.

\section{Wino at the $\mathrm{LHC}$}

Now we are at the position to discuss how we can test the "no singlet scenario" at the LHC. In studying superparticles at the LHC, it is often assumed that the productions of superparticles are mostly via productions of colored superparticles. Even though the primary superparticles are scalar quarks and/or gluino, they decay into various lighter superparticles which may be scalar leptons, charginos, and/or neutralinos. Of course, those processes are important when scalar quarks and/or gluino are not too heavy.

Since we are interested in the case where all the sfermions (as well as heavy Higgs multiplets) have masses of order $100 \mathrm{TeV}$, they are irrelevant for the LHC. Hereafter, we will not consider the production of those particles.

On the contrary, masses of gauginos (in particular, that of gluino) are of order 100 $\mathrm{GeV}-1 \mathrm{TeV}$. As discussed in the previous section, the gluino-to-Wino mass ratio is a free parameter in the "no singlet scenario", since the prediction of pure anomaly mediation, which gives $M_{3} / M_{2} \simeq 8$, can be significantly altered. For a given Wino mass $M_{2}$, the discovery of SUSY at the LHC depends on the ratio $M_{3} / M_{2}$.

When $M_{3} / M_{2}$ is relatively small, the gluino is light enough so that a significant amount of gluino is produced at the LHC. In this case, the dominant production processes of superparticles are gluino pair productions. The primary gluinos decay into lighter superparticles and, at the end of decay chain, the neutral Winos are produced. Consequently, we observe events with energetic jets and large missing $E_{T}$. In this case, signals from the production of superparticles are basically the same as those in well-studied SUSY models.

Hereafter, we concentrate on a rather pessimistic case where the gluino mass is so large

\footnotetext{
${ }^{3}$ See the related calculation of the branching ratios for a nearly degenerate heavy lepton pair in Ref. [17.
} 
that a gluino production rate is suppressed at the LHC. In this case, the only possibility of detecting superparticles is direct productions of charged and neutral Winos. (Notice that the Bino production cross section is very small since the scalar quarks are extremely heavy.)

In our case, the neutral Wino is the LSP and hence is stable. Thus, even if it is produced at the LHC, it does not leave any consequence on the detector. The charged Wino is heavier than the neutral one and hence is unstable. The mass difference $\Delta M=$ $m_{\vec{W}^{ \pm}}-m_{\vec{W}^{\text {o }}}$ is expected to be $160-170 \mathrm{MeV}$, and the charged Wino mainly decays as $\tilde{W}^{ \pm} \rightarrow \tilde{W}^{0} \pi^{ \pm}$with lifetime of $\mathcal{O}\left(10^{-10}\right)$ sec. Thus, most of charged Winos decay before it reaches a muon detector. In addition, the emitted pion has very tiny energy; its boost factor is typically $\gamma_{\pi} \sim \mathcal{O}(1)$. Thus, it is challenging to identify such a low-energy pion. These facts make the discover of Wino production events very difficult.

Winos can be pair-produced at the LHC via Drell-Yang processes $q \bar{q} \rightarrow \tilde{W}^{+} \tilde{W}^{-}$and $q \bar{q}^{\prime} \rightarrow \tilde{W}^{ \pm} \tilde{W}^{0}$. However, these events cannot be recorded since there is no trigger relevant for them. Indeed, in order for the event to be recorded, the final state should contain high energy jet(s) and/or high energy electro-magnetic activity. In the Drell-Yang process, we do not expect such activities since the charged Winos decay before reaching the muon detector.

However, events may be recorded if Winos are produced with high $E_{T}$ jet(s). In particular, ATLAS [18] and CMS [19] both plan to trigger on missing $E_{T}$ events with energetic jets; if the transverse energy of the jet and missing $E_{T}$ are both larger than 50 - $100 \mathrm{GeV}$, events will be recorded. (Hereafter, we call such a trigger as J+XE trigger.)

With $p p$ collision at the LHC, a Wino pair can be produced in association with a high $E_{T}$ jet via the following parton-level processes:

$$
\begin{aligned}
& q \bar{q} \rightarrow \tilde{W}^{+} \tilde{W}^{-} g, \quad g q \rightarrow \tilde{W}^{+} \tilde{W}^{-} q, \quad g \bar{q} \rightarrow \tilde{W}^{+} \tilde{W}^{-} \bar{q} \\
& q \bar{q}^{\prime} \rightarrow \tilde{W}^{ \pm} \tilde{W}^{0} g, \quad g q \rightarrow \tilde{W}^{ \pm} \tilde{W}^{0} q^{\prime}, \quad g \bar{q} \rightarrow \tilde{W}^{ \pm} \tilde{W}^{0} \bar{q}^{\prime} .
\end{aligned}
$$

Gluon and (anti-)quark in the final state are hadronized and become energetic jets. Thus, at the trigger level, this class of events are characterized by a high $E_{T}$ mono-jet, missing 


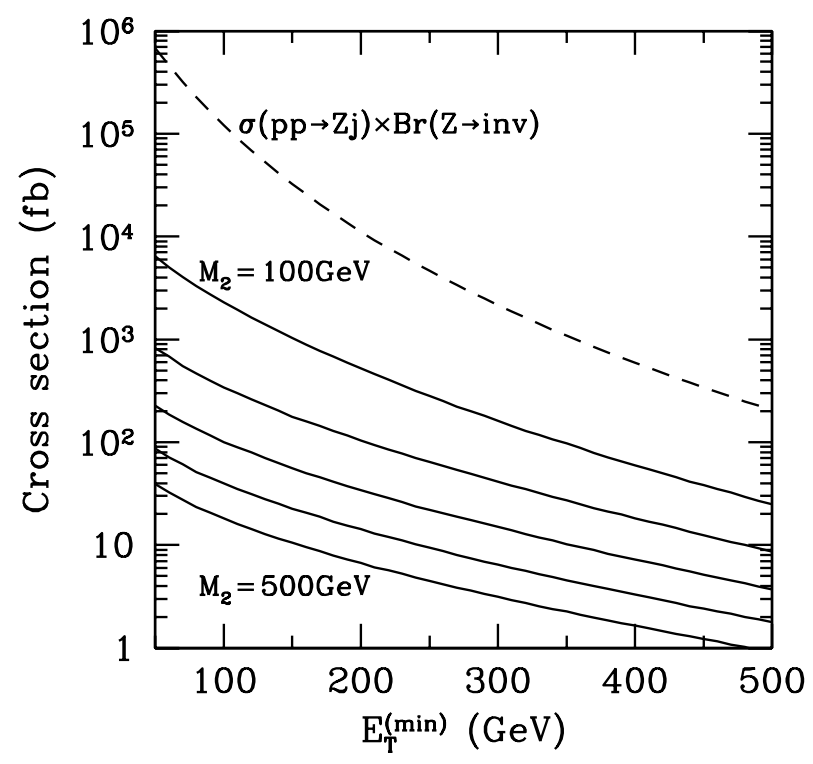

Figure 2: Total cross section of a Wino pair + mono-jet production event $\sigma(p p \rightarrow$ $\left.\tilde{W}^{+} \tilde{W}^{-} j\right)+\sigma\left(p p \rightarrow \tilde{W}^{ \pm} \tilde{W}^{0} j\right)$ at $\sqrt{s}=14 \mathrm{TeV}$ as a function of $E_{T}^{(\min )}$ (solid). $M_{2}$ is taken to be 100,200,300, 400, and $500 \mathrm{GeV}$ from above. Cross section of the process $p p \rightarrow Z j$ multiplied by the invisible branching ratio of $Z$ is also plotted (dashed).

$E_{T}$, and no other energetic activity, since the charged Wino decays before reaching the muon detector.

In order to estimate the total cross section of the Wino pair + mono-jet production process, we calculate the cross section of the processes (11) and (12). We require that the transverse energy of mono-jet be larger than $E_{T}^{(\min )}$, and calculate the cross section as a function of $E_{T}^{(\min )}$. In our calculation, $E_{T}$ of jet is approximated to be the same as that of the final-state gluon or (anti-)quark. We have used the CTEQ6L parton distribution function [21]. The result is shown in Fig. 2] ${ }^{4}$ With the luminosity of $\mathcal{O}(10-100) \mathrm{fb}^{-1}$, sizable numbers of Wino production events are expected even if we require that the associated jet has transverse energy of $\mathcal{O}(100) \mathrm{GeV}$.

Once the events are recorded, the processes $p p \rightarrow \tilde{W}^{+} \tilde{W}^{-} j$ and $p p \rightarrow \tilde{W}^{ \pm} \tilde{W}^{0} j$ (with $j$ being jet) may provide distinguishable signals of the Wino LSP scenario at off-line analysis. One possible signal is the track of charged Wino, as also suggested for the study

\footnotetext{
${ }^{4}$ We have cross checked our results by using the codes, FeynArts [22] and FormCalc [23].
} 
of Tevatron [11]. If the charged Wino travels $\mathcal{O}(10) \mathrm{cm}$ or longer before it decays, it hits some of the detectors and the track from charged Wino may be reconstructed. The track from charged Wino may be also distinguished from tracks from other standard-model charged particles by using the time-of-flight information and/or by measuring ionization energy loss rate $(d E / d x)$.

In addition, it is important to note that, with the lifetime of $\mathcal{O}\left(10^{-10}\right)$ sec, most of the charged Winos are likely to decay inside the detector. Then, we may observe tracks which disappear somewhere in the detector. This will be regarded as a spectacular signal of new physics beyond the standard model. In particular, in the ATLAS detector, the transition radiation tracker (TRT) will be implemented, which is located at $56-107 \mathrm{~cm}$ from the beam axis [20]. The TRT continuously follows charged tracks. Thus, if the charged Wino decays inside the TRT, the charged Wino track will be identified by off-line analysis. In addition, with the TRT, charged pions emitted by the decays of $\tilde{W}^{ \pm}$may be also seen. Such events have very rare backgrounds and hence they can be used for the discovery of the Wino production.

We calculate the cross section of the processes $p p \rightarrow \tilde{W}^{+} \tilde{W}^{-} j$ and $p p \rightarrow \tilde{W}^{ \pm} \tilde{W}^{0} j$, with the requirement that at least one charged Wino travels transverse length $L_{T}$ longer than $L_{T}^{(\min )}$ before it decays. At the trigger level, no hadronic nor electro-magnetic activities will be identified except for the mono-jet, so the missing $E_{T}$ is equal to the transverse energy of the mono-jet. In order to use the $\mathrm{J}+\mathrm{XE}$ trigger, we impose a cut such that $E_{T} \geq 100 \mathrm{GeV}$ for the final-state jet. $L_{T}$ is sensitive to the decay width of charged Wino. Since we consider the case where gauginos are the only light superparticles, we use Eq. (9) for $\Delta M$. The decay width of charged Wino is given in Eq. (10).

Cross sections for several values of $L_{T}^{(\min )}$ are shown in Fig. [3] Assuming $\mathcal{L} \sim 100 \mathrm{fb}^{-1}$ for high luminosity run of the LHC, for example, a sizable number of charged Winos with $L_{T} \sim \mathcal{O}(10) \mathrm{cm}$ will be produced. Thus, if we can find their tracks, it will provide an intriguing signal which cannot be explained in the standard model. Requiring 10 events with $L_{T} \geq 50 \mathrm{~cm}(1 \mathrm{~m})$ with $\mathcal{L}=100 \mathrm{fb}^{-1}$, for example, the LHC can cover $M_{2} \leq 350$ $\mathrm{GeV}(200 \mathrm{GeV})$. Thus, the search for (short) tracks of heavy charged particles is strongly 


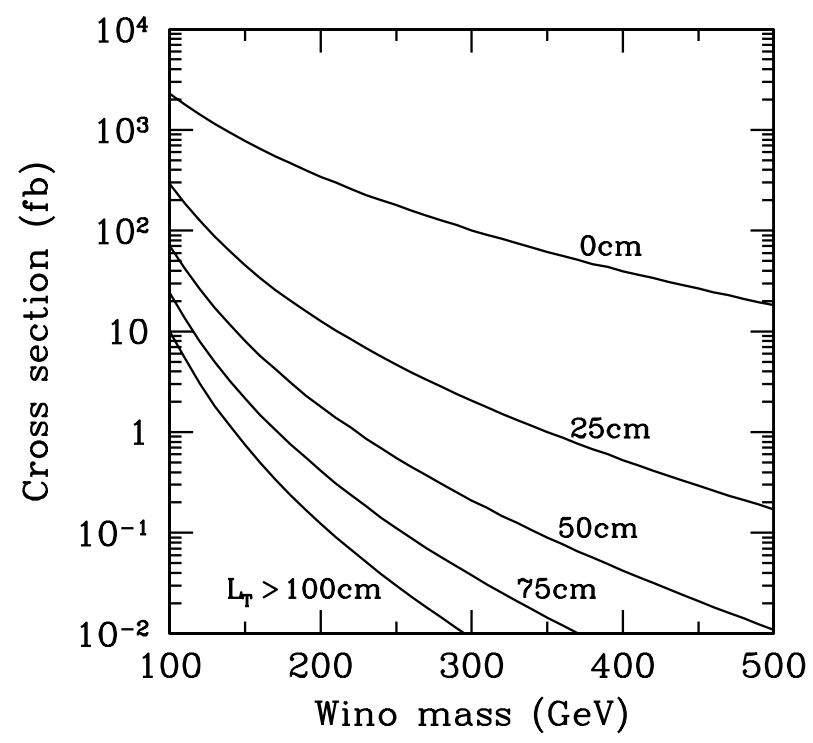

Figure 3: Cross section of the process $p p \rightarrow \tilde{W} \tilde{W} j$ at $\sqrt{s}=14 \mathrm{TeV}$ as a function of $M_{2}$. $E_{T}$ of the jet is required to be larger than $100 \mathrm{GeV}$, and the minimum value of $L_{T}$ is 0 , $25,50,75$, and $100 \mathrm{~cm}$.

suggested when other signals from superparticles cannot be discovered at the LHC.

Even without looking for tracks of $\tilde{W}^{ \pm}$, an excess of mono-jet events with large missing $E_{T}$ may provide another signal of the "no singlet scenario". If no special effort is made to find Wino tracks, Wino production events look like mono-jet events with missing $E_{T}$ (assuming that Winos decay with $L_{T} \lesssim 1 \mathrm{~m}$ or so). Detailed study of such events may give another test of the Wino production.

The most serious standard-model background for the mono-jet event is from the process $p p \rightarrow Z j$ followed by $Z \rightarrow \nu \bar{\nu}$. At the parton level, $Z j$ production is from $q \bar{q} \rightarrow Z g$, $g q \rightarrow Z q$, and $g \bar{q} \rightarrow Z \bar{q}$. In order to estimate the number of backgrounds, we calculate the cross section of these processes as functions of $E_{T}$ of the jet. The total cross section of $p p \rightarrow Z j$ multiplied by $\operatorname{Br}(Z \rightarrow \nu \bar{\nu})$ is also plotted in Fig. 2. The background cross section is several orders of magnitude larger than the signal cross section. Importantly, however, the cross section of the process $p p \rightarrow Z j$ will be well understood by using the decay mode $Z \rightarrow l^{+} l^{-}$(with $l^{ \pm}$being charged lepton). We expect that the charged lepton pair from $Z$ can be identified and hence $\sigma(p p \rightarrow Z j)$ will be determined with high 
precision.

In order to perform a statistical analysis of mono-jet + missing $E_{T}$ event, it is crucial to understand the total background cross section. As well as the process $p p \rightarrow Z j$, other processes may contribute to the background. One of such is the process $p p \rightarrow Z Z j$ if both $Z$ 's decay invisibly. Thus, we have also calculated the cross section of this process. Taking account of the branching ratio of the invisible mode of $Z$, we have found that the background cross section from $p p \rightarrow Z Z j$ is $29-0.23$ fb for $E_{T}=100-500 \mathrm{GeV}$, which is smaller than the signal cross section when the Wino mass is smaller than $\sim 500$ $\mathrm{GeV}$. In addition, we will obtain some information about this class of backgrounds using leptonic decay modes of $Z$. Thus, we consider that the process $p p \rightarrow Z Z j$ is not a serious background. The process $p p \rightarrow W^{ \pm} j$ followed by leptonic decay of $W^{ \pm}$may also give backgrounds if the charged lepton goes into the beam pipe. However, the probability of charged lepton escaping into the beam pipe may not be so large. In addition, the number of this type of backgrounds will be also estimated by studying mono-jet events with a single charged lepton and missing $E_{T}$. We consider that cross sections of other processes resulting in the mono-jet + missing $E_{T}$ final state are also small enough to be neglected.

If the standard-model cross section of the mono-jet + missing $E_{T}$ event is understood precisely, an excess beyond the statistical error may be regarded as a signal of Wino + mono-jet events. That is, the expected number of background events is $\mathcal{O}\left(10^{7}-10^{4}\right)$ for $E_{T}>100-500 \mathrm{GeV}$ of the mono-jet at a high luminosity $L=100 \mathrm{fb}^{-1}$, for example. Then, the number of the Wino production events with a mono-jet becomes statistically significant when $M_{2} \lesssim 400 \mathrm{GeV}$ (see Fig. 2).

It may be the case that the background cross section cannot be determined with such a high precision because of some systematic errors. We will not go into the detailed study of systematic error. Instead, we estimate how well the systematic error should be controlled to find an anomaly; in order to cover the Wino mass of 100, 200, 300, 400, and $500 \mathrm{GeV}$, systematic error in the determination of background cross section should be smaller than $1.9,0.28,0.083,0.032$, and $0.015 \%(12,4.2,1.8,0.87$, and $0.45 \%)$ for $E_{T}^{(\min )}>100 \mathrm{GeV}$ $(500 \mathrm{GeV})$. 
Finally, let us briefly discuss what happens if Higgsino mass (i.e., the $\mu$-parameter) is smaller than $\sim \mathrm{TeV}$. So far, we have considered the case where superparticles other than gauginos are as heavy as $\mathcal{O}(100) \mathrm{TeV}$, and hence the mass difference between charged and neutral Winos is determined mainly by the radiative corrections from gauge-boson loops. In this case, $\Delta M$ is given by Eq. (9) and the lifetime of charged Wino is more or less predicted. If the $\mu$-parameter is smaller, on the contrary, $\Delta M$ is affected by the gaugino-Higgsino mixing. In this case, $\Delta M$ can be enhanced or suppressed, depending on $\mu$ - and other parameters. In particular, $\Delta M$ becomes smaller than the pion mass and the lifetime of charged Wino becomes longer in some parameter region. In this case, it is much easier to find tracks of the charged Wino since $\tilde{W}^{ \pm}$does not decay inside the detector. It should be also noticed here that such a charged Wino with the lifetime of $\mathcal{O}\left(10^{-8}\right)$ sec may be used as a probe of deep interior of heavy nuclei [24].

\section{Acknowledgment}

The authors would like thank S. Asai, J. Kanzaki and T. Kobayashi for valuable discussion and useful comments. M.I. is grateful to T. Hahn and C. Schappacher for kind explanation of FormCalc. This work was supported in part by the U.S. Department of Energy under contract number DE-AC02-76SF00515 (M.I.) and by the Grant-in-Aid for Scientific Research from the Ministry of Education, Science, Sports, and Culture of Japan, No. 15540247 (T.M.) and 14102004 (T.T.Y.).

\section{References}

[1] H. P. Nilles, Phys. Rept. 110 (1984) 1; S. P. Martin, arXiv:hep-ph/9709356.

[2] L. Randall and R. Sundrum, Nucl. Phys. B 557, 79 (1999).

[3] G. F. Giudice, M. A. Luty, H. Murayama and R. Rattazzi, JHEP 9812, 027 (1998).

[4] F. Gabbiani, E. Gabrielli, A. Masiero and L. Silvestrini, Nucl. Phys. B 477, 321 (1996). 
[5] W. M. Yao et al. [Particle Data Group], J. Phys. G 33, 1 (2006).

[6] S. Weinberg, Phys. Rev. Lett. 48 (1982) 1303; See, for a recent work, K. Kohri, T. Moroi and A. Yotsuyanagi, Phys. Rev. D 73 (2006) 123511, and references therein.

[7] M. Fukugita and T. Yanagida, Phys. Lett. B 174, 45 (1986); For a recent review, W. Buchmuller, R. D. Peccei and T. Yanagida, Ann. Rev. Nucl. Part. Sci. 55, 311 (2005).

[8] M. Ibe, R. Kitano, H. Murayama and T. Yanagida, Phys. Rev. D 70, 075012 (2004);

M. Ibe, R. Kitano and H. Murayama, Phys. Rev. D 71, 075003 (2005).

[9] T. Moroi and L. Randall, Nucl. Phys. B 570, 455 (2000).

[10] M. Ibe, Y. Shinbara and T. T. Yanagida, arXiv hep-ph/0608127.

[11] J. L. Feng, T. Moroi, L. Randall, M. Strassler and S. f. Su, Phys. Rev. Lett. 83, 1731 (1999).

[12] M. Kawasaki, F. Takahashi and T. T. Yanagida, Phys. Lett. B 638, 8 (2006); M. Kawasaki, F. Takahashi and T. T. Yanagida, Phys. Rev. D 74, 043519 (2006); M. Endo, M. Kawasaki, F. Takahashi and T. T. Yanagida, arXiv hep-ph/0607170.

[13] J. D. Wells, Phys. Rev. D 71, 015013 (2005).

[14] K. Inoue, M. Kawasaki, M. Yamaguchi and T. Yanagida, Phys. Rev. D 45, 328 (1992).

[15] T. Gherghetta, G. F. Giudice and J. D. Wells, Nucl. Phys. B 559, 27 (1999).

[16] A. Heister et al. [ALEPH Collaboration], Phys. Lett. B 533, 223 (2002).

[17] S. D. Thomas and J. D. Wells, Phys. Rev. Lett. 81, 34 (1998).

[18] ATLAS homepage, http://atlas.web.cern.ch.

[19] CMS homepage, http://cms.cern.ch.

[20] ATLAS Collaboration, CERN-LHCC-97-16; CERN-LHCC-97-17.

[21] J. Pumplin, D. R. Stump, J. Huston, H. L. Lai, P. Nadolsky and W. K. Tung, JHEP 0207, 012 (2002). 
[22] T. Hahn, Comput. Phys. Commun. 140, 418 (2001).

[23] T. Hahn and M. Perez-Victoria, Comput. Phys. Commun. 118, 153 (1999).

[24] K. Hamaguchi, T. Hatsuda and T. T. Yanagida, arXiv:hep-ph/0607256. 